\title{
Determination of enantiomeric composition of fluoxetine by synchronous fluorescence spectrometry coupled with multivariate calibration in biological samples
}

\author{
Roman Poláček, Pavel Májek, Jana Sádecká \\ Institute of Analytical Chemistry, Faculty of Chemical and Food Technology, Slovak University of Technology, \\ Radlinského 9, SK-812 37 Bratislava, Slovakia \\ roman.polacek@stuba.sk
}

\begin{abstract}
This paper presents rapid and low cost analytical method for the determination of the fluoxetine enantiomeric composition in biological samples (urine). The combination of synchronous fluorescence spectrometry and inverse multivariate calibration methods was used. The chiral recognition of the fluoxetine was based on the creating of the diastereomeric complexes with $\beta$-cyclodextrin. A net analytical signal of diastereomeric complexes was obtained by the addition of aliquot part of urine into calibration and validation sets. This step ensures the elimination of the urine matrix effect. The synchronous fluorescence spectra at the constant wavelength differences $(\Delta \lambda)$ of 30 and $50 \mathrm{~nm}$, based on RMS \%RE values, were chosen for chemometric analysis. Principal component regression (PCR) and partial least square method (PLS) were compared to determine the enantiomeric composition. The most suitable results were provided by the PLS model constructed from the synchronous data at $\Delta \lambda=50 \mathrm{~nm}$. The calculated figure of merit was used for validation of proposed method.
\end{abstract}

Keywords: chiral analysis, fluoxetine enantiomers, synchronous fluorescence spectrometry, multivariate calibration, biological samples

\section{Introduction}

Chiral analysis is traditionally performed by chiroptical methods, which use interactions between the stereogenic center of the chiral molecules and polarized light. These methods are polarimetry, optical rotatory dispersion, Raman optical activity and circular dichroism (Horvát et al., 1997; Gergely, 2000; Konno et al., 2013; Shen et al., 2014). Nonchiroptical methods include separation technique such as chromatography (gas or liquid) or capillary electrophoresis (Mohr et al., 2012; Suliman and Elbashir, 2012; Douša et al., 2013). Modern trends in non-chiroptical methods are represented by the combination of molecular spectrometry and inverse multivariate calibration methods.

The chiral analysis, performed by non-chiroptical methods, needs formation of diastereomeric complex for the chiral recognition. This complex is created by the interaction between the stereogenic center of the optically active molecules and the chiral center of selector (Sullivan, 1978; Finn, 2012). Cyclodextrins (CDs) belong to widely used chiral selectors. CDs are cyclic oligosaccharides molecule with a hydrophilic outer surface and a hydrophobic inner cavity made up of six $(\alpha-\mathrm{CD})$, seven $(\beta$-CD) and eight $(\gamma$-CD) glucose units. The CD cavity provides non-covalent interactions with the chiral analyte, which is frequently used in molecular spec- trometry with chemometric processing. Published works have been summarized recently (Poláček and Májek, 2016). The chemometric analysis, namely multivariate calibration (MVC) methods, are able to evaluate the small changes between inclusion complexes of analytes. In molecular spectrometry, inverse MVC methods are used extensively because they allow a large number of predictor variables and tolerate correlation between predictor variables in the original measured spectral data (Wise et al., 2006). The inverse multivariate regression models, adopt in this study, are PCR and PLS which are usually compared because they have different means of creation working matrix from original data. PGR only considers the spectral data into the transformation process, while PLS actively involves both spectral and concentration data in performing the transformation [16]. More details about PCR and PLS were referred in (Wold et al., 2001; Wise et al., 2006; Rajalahti and Kvalheim, 2011) and are not described in this paper.

Fluoxetine, $\quad(( \pm)-N$-methyl- $\gamma$-[4-(trifluoromethyl) phenoxy] benzene propanamine) (FLX), is a third generation of stereospecific antidepressant drugs, which selective inhibit serotonin in neurons. It could have very high therapeutic and commercial potential because it cured mental depression, obsessive-compulsive disorder, nervous bulimia, and premenstrual dysphoric disorder. (S)-FLX ex- 
hibited a longer duration of action and is approximately 1.5 times more potent than $R$-enantiomer and displays a threefold longer duration of action (Grodner and Sitkiewicz, 2013).

Chromatographic methods, as LC or GC with chiral columns or chiral additives into mobile phase are typical for the determination of the FLX enantiomers (Lerena et al, 2003; Li et al., 2004; Mifsud and Sqhendo, 2012; Yu et al., 2012; Ribeiro et al., 2013). Few studies of FLX chiral analysis using non-chromatography methods such as capillary electrophoresis were published (Asensi-Bernardi et al., 2013) and NMR (Ali et al., 2005; Shamsipur et al., 2007).

Our research presents the feasibility of FLX chiral analysis by combination of synchronous fluorescence spectrometry and inverse MVC methods (PCR and PLS) which were compared to obtain the best resolution of the FLX enantiomers in complicate biological matrix such as urine. For chiral recognition in analytes the diastereomeric complexes were created by $\beta$-CD which was chosen in our previously study (Poláček and Májek, 2015).

\section{Materials and methods}

\section{Reagents and samples}

All experiments were performed by analytical reagent grade chemicals and doubly distilled water. Enantiomers stock solutions with concentration $10 \mathrm{~mol} \mathrm{~L}^{-1}$ in water were prepared. The stock solution of the $\beta$-CD was prepared daily by dissolving $0.57 \mathrm{~g}$ into $50 \mathrm{~mL}$ water in a volumetric flask. All chemicals were pursed in Sigma-Aldrich (USA).

Biological samples (adult man and women urine) were obtained in the morning. It was assumed that fluoxetine was absent in these samples.

\section{Apparatus}

Lumina fluorescence spectrometer (Thermo Scientific) with 150 Watt Ozone free Xenon lamp was used for obtain fluorescence spectra. The scan speed was $200 \mathrm{~nm} \mathrm{~min}{ }^{-1}$ and both splits were set at $5.0 \mathrm{~nm}$. Synchronous fluorescence spectra with constant wavelength differences $(\Delta \lambda)$ were measured by simultaneous scanning the excitation and emission monochromators in the emission wavelength range at $250-350 \mathrm{~nm}$. The $\Delta \lambda$ was set from 10 to $100 \mathrm{~nm}$, with step $5 \mathrm{~nm}$ between them. The measurements were performed in triplicate at ambient temperature using a $1.0 \mathrm{~cm}$ quartz cell with excitation and emission bandpass set at $0.1 \mathrm{~nm}$. The spectral data were recorded by LUMINOUS SOFTWARE. All calculations were done using Microsoft Office Excel 2010, Statistica version 12.0 (StatSoft, USA, 2013), MATLAB 8.1 (The MathWorks Inc., USA,
2013) and PLS_Toolbox version 7.9 (Eigenvector Research Inc., USA, 2014).

The absorbance was monitored by UV-1800 Spectrophotometer (Shimadzu, Japan).

\section{Sample preparation}

Urine samples were treated by pipetting the aliquot parts of urine into $50 \mathrm{~mL}$ flask, where they were spiked with appropriate amounts of standard solutions of both enantiomers and filled with water to the mark. The mole fraction of the $(S)$-FLX in urine, within the range from 60 to $90 \%$, was determined as described in (Yee et al., 2000). From these solutions $3.8 \mathrm{~mL}$ was pipetted into $10 \mathrm{~mL}$ flask, adding the proper aliquot of the stock solutions of the chiral selector and was made up with water. The test solutions were put into dark for $10 \mathrm{~min}$ and analysed. All measurements were performed triplicate.

\section{Analytical figure of merit}

Figure of merit characterizes a quality of multivariate calibration models built on spectral data by PCR and PLS methods. This process use a net analyte signal (NAS) calculations defined as the part of measured signal that is unique for the consider enantiomer. The estimation of the figure of merit in PCR and PLS calibration models is enabled by NAS using descripted equations at earlier study (Ferré and Faber, 2003) and are not written in detail here.

\section{Results and discussion}

\section{Selection of optimum $\Delta \lambda$}

The synchronous fluorescence technique requires optimization of $\Delta \lambda$ value due to effect on the shape of the spectrum, signal value and acquisition of the good selectivity and sensitivity. $\Delta \lambda$ values are selected empirically and the fluorescence intensity must be taken into account. The maximum of fluorescence intensity for both enantiomers was in the range of $\Delta \lambda=20-60 \mathrm{~nm}$. Outside this interval the intensity decreases. Inclusions complexes of both enantiomers provide the spectra with small changes between them. Therefore, the synchronous spectral data without MVG is not possible to evaluate. PCR and PLS calibration models were constructed using the synchronous spectra at $\Delta \lambda$ in the interval 20-60 $\mathrm{nm}$. The root-mean-square percent relative error (RMS \% RE) was used for finding the suitable model, built on the synchronous spectral data.

RMS \% RE is a useful for revealing the ability of the regression model to accurately predict the enantiomeric composition of the validation samples. RMS \% RE is given by: 


$$
\begin{gathered}
R M S \% R E=\sqrt{\frac{\sum\left(\% R E_{i}\right)^{2}}{n} ;} \\
\% R E_{i}=\frac{100 \times\left(\breve{\mathrm{y}}_{i}-\mathrm{y}_{i}\right)}{\mathrm{y}_{i}}
\end{gathered}
$$

where $y_{i}$ is the experimentally observed result for the $i$-th validation sample, $\hat{y}_{i}$ is the predicted result, and $n$ is the number of validation sample in the set (Poláček et al., 2016).

The values of RMS \%RE from PCR and PLS calibration models for urine samples are shown in Fig. 1. The lower values for (S)-FLX indicated that $(S)$-FLX has stronger interaction with $\beta$-CD in comparison with $(R)$-FLX. The most suitable values of RMS \%RE are obtained at $\Delta \lambda=30$ and $50 \mathrm{~nm}$. Comparison of the RMS \%RE obtained by PCR and PLS models indicates that PLS models are slightly more suitable to prediction the enantiomeric composition.

\section{Investigation of matrix effect (determination in urine)}

The matrix effect is the difference between the net fluorescence signal of an analyte in standard solution and the response for the same analyte at the same concentration in a biological matrix (urine).
Human urine contains a large number of fluorophores which can fluorescence under UV excitation and may influence the determination (Kusnir, 2005).

Synchronous fluorescence spectra for pure analytes and urine were collected and possible matrix effect of urine on enanatiomeric recognition was appreciated. Pure urine samples show different fluorescence intensity at various $\Delta \lambda$. In the ranges of $\Delta \lambda 10-35 \mathrm{~nm}$ and $65-100 \mathrm{~nm}$ only one peak is recorded in spectra but in the interval $\Delta \lambda=40-60 \mathrm{~nm}$ two peaks are presented.

Complex of $(R)$-FLX with $\beta$-CD shows one maximum at $295 \mathrm{~nm}$ and the complex of (S)-FLX with $\beta$-CD has two maxima at 295 and $320 \mathrm{~nm}$ at $\Delta \lambda=30 \mathrm{~nm}$. In the case $\Delta \lambda=50 \mathrm{~nm}$ both of the complexes show two maximal values of the fluorescence intensity at 280 and $305 \mathrm{~nm}$, respectively. The fluorescence maxima of the pure urine are at $315 \mathrm{~nm}$ when $\Delta \lambda=30 \mathrm{~nm}$ and at $340 \mathrm{~nm}$ when $\Delta \lambda=50 \mathrm{~nm}$ (Fig. 2). Given the potential effect of urine matrix on the determination of enantiomeric composition, urine was included as separate component into calibration and validation sets.

The differences in the fluorescence intensities of both inclusion complexes of FLX enantiomers with $\beta$-CD are sufficient to reflect composition changes in solutions which contain the various ratios of enantiomers.

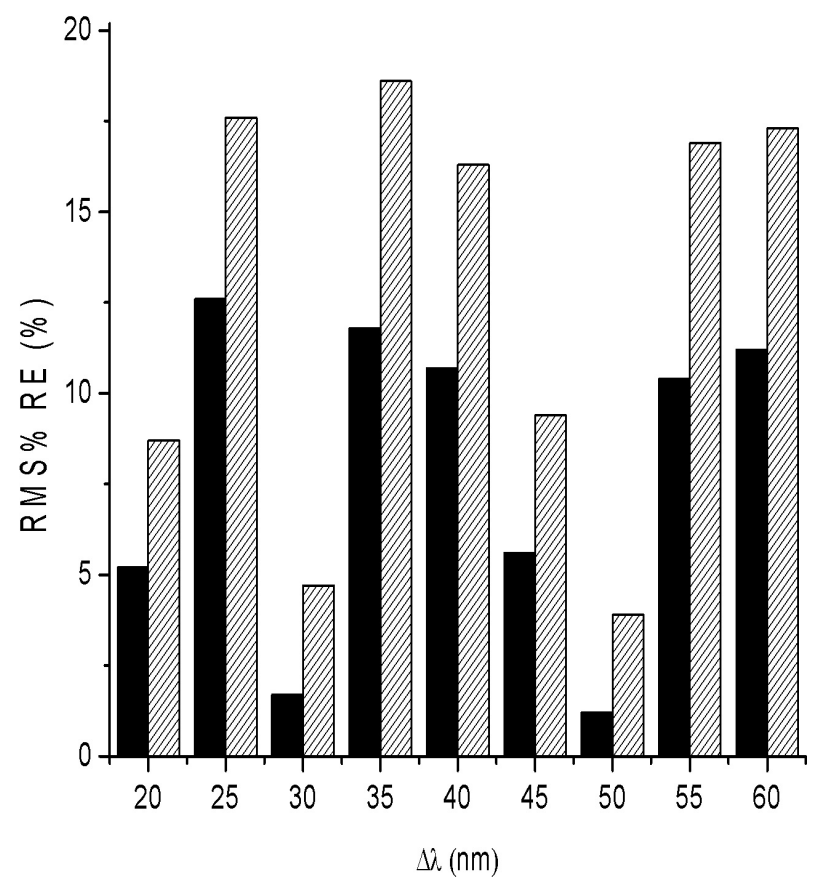

A

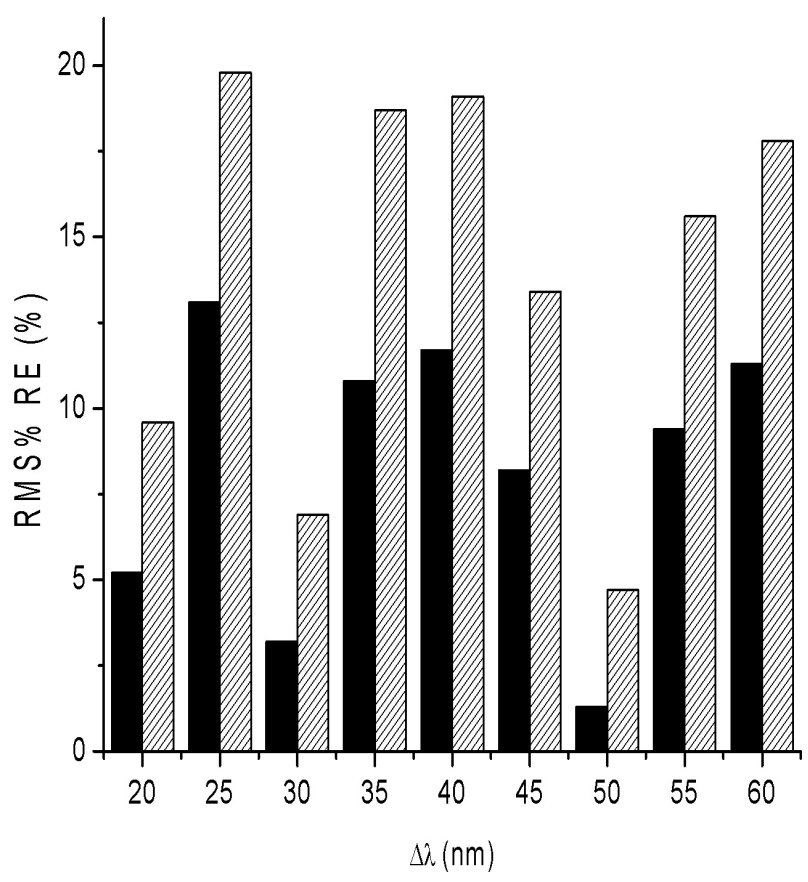

B

Fig. 1. The RMS \%RE values obtained for (S)-FLX (black) and (R)-FLX (cross-hatched) from PCR(A) and PLS (B) calibration models. 


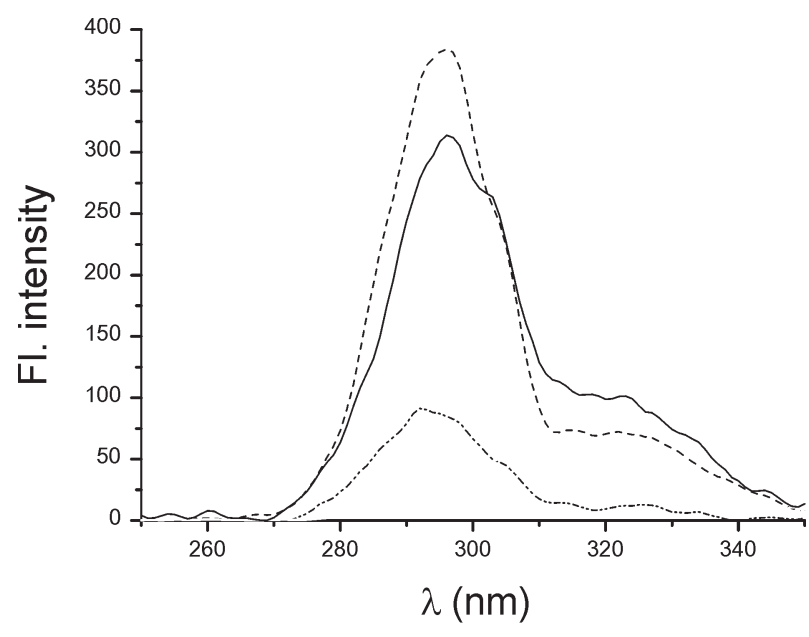

A

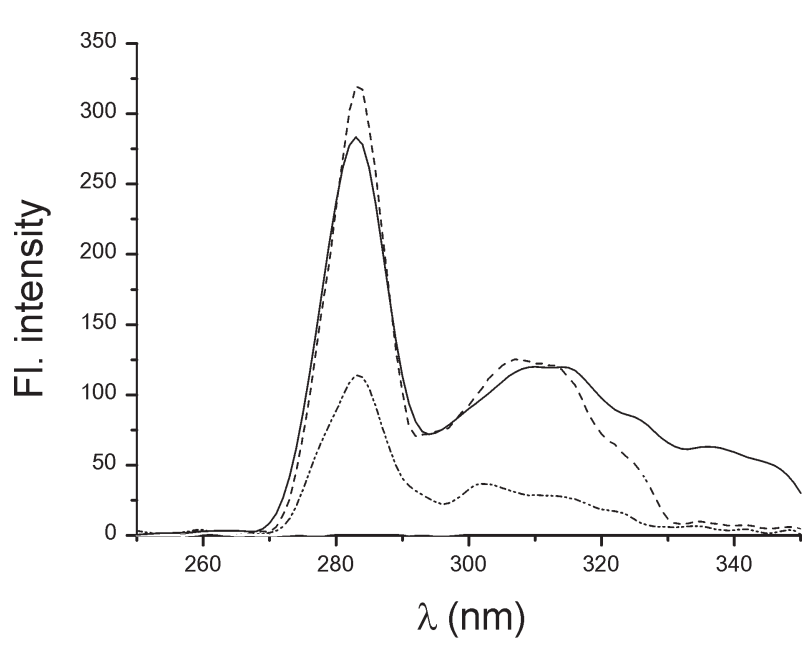

B

Fig. 2. The synchronous fluorescence spectra at $\Delta \lambda=30 \mathrm{~nm}$ (A) and at $\Delta \lambda=50 \mathrm{~nm}$ (B) for (S)-FLX (dash line); (R)-FLX (dash dot dot line); pure urine (gray line) and spiked urine sample (black line).

Tab. 1. Figure of merit and predicted values of PCR models for determination of FLX enantiomeric composition in biological (urine) sample.

\begin{tabular}{|c|c|c|c|c|c|c|}
\hline \multirow{3}{*}{ samples } & \multicolumn{4}{|c|}{ mole fraction $(\%)$} & & \\
\hline & \multicolumn{2}{|l|}{ spike (\%) } & \multicolumn{2}{|c|}{$\Delta \lambda=30 \mathrm{~nm}$} & \multicolumn{2}{|c|}{$\Delta \lambda=50 \mathrm{~nm}$} \\
\hline & $(S)$-FLX & $(R)$-FLX & $(S)$-FLX & $(R)$-FLX & $(S)$-FLX & $(R)$-FLX \\
\hline 1 & 0.867 & 0.133 & 0.858 & 0.142 & 0.869 & 0.131 \\
\hline 2 & 0.760 & 0.240 & 0.770 & 0.230 & 0.767 & 0.233 \\
\hline 3 & 0.640 & 0.360 & 0.664 & 0.336 & 0.645 & 0.355 \\
\hline \multirow[t]{9}{*}{4} & 0.827 & 0.173 & 0.822 & 0.178 & 0.832 & 0.168 \\
\hline & \multicolumn{2}{|c|}{ Number of latent variables } & 15 & 15 & 11 & 11 \\
\hline & Accuracy & RMSEC $^{\mathbf{a}}$ & 0.733 & 0.733 & 0.879 & 0.879 \\
\hline & & RMSECV $^{b}$ & 0.687 & 0.687 & 0.795 & 0.795 \\
\hline & & RMSEP $^{c}$ & 0.567 & 0.567 & 0.650 & 0.650 \\
\hline & & RMS \% RE $\mathbf{E}^{\mathbf{d}}$ & 2.1 & 5.2 & 0.7 & 2.2 \\
\hline & \multicolumn{2}{|l|}{ Sensitivity } & 1.2 & 1.2 & 1.7 & 1.7 \\
\hline & \multicolumn{2}{|c|}{ Analytical sensitivity $^{-1}$} & 0.6 & 0.2 & 2.4 & 0.8 \\
\hline & \multicolumn{2}{|c|}{$\mathbf{R}^{2}$ Prediction } & 0.992 & 0.992 & 0.997 & 0.997 \\
\hline
\end{tabular}

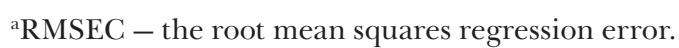

${ }^{\mathrm{b}} \mathrm{RMSECV}$ - the root mean squares regression error of cross-validation.

${ }^{\mathrm{c}} \mathrm{RMSEP}$ - the root mean squares regression error of prediction.

${ }^{\mathrm{d}} \mathrm{RMS} \% \mathrm{RE}-$ the root mean squares percent relative error.

Determination of enantiomeric composition in urine The PCR and PLS calibration models were used to determine the enantiomeric composition in urine samples. The choice of the most suitable regression was performed by comparing the performance prediction of the model characteristics. The comparison was based on the root mean square error of the calibration (RMSEC), the root mean square error of cross-validation (RMSECV), the root mean square error of prediction (RMSEP) and RMS \% RE. Results for the figure of merit for PCR models and PLS models are presented in Tables 1 and 2 . Obtained values have good prediction abilities to determine enantiomeric composition. RMSEP values of all PLS models are closed to 1 what means that models can predict enantiomeric composition by less than $1 \%$ from each enantiomer in racemic mixture. The determined values of RMS \% RE for PCR models are worse than for PLS models. In comparison to regression methods, higher values for the $(R)$-FLX enantiomer was achieved than for $(S)$-FLX. The PCR models require large numbers 
Tab. 2. Figure of merit and predicted values of PLS models for determination of FLX enantiomeric composition in biological (urine) sample.

\begin{tabular}{|c|c|c|c|c|c|c|}
\hline \multicolumn{7}{|c|}{ mole fraction $(\%)$} \\
\hline \multirow{2}{*}{ samples } & \multicolumn{2}{|l|}{ spike (\%) } & \multicolumn{2}{|c|}{$\Delta \lambda=30 \mathrm{~nm}$} & \multicolumn{2}{|c|}{$\Delta \lambda=50 \mathrm{~nm}$} \\
\hline & $(S)$-FLX & $(R)$-FLX & (S)-FLX & $(R)$-FLX & $(S)$-FLX & $(R)$-FLX \\
\hline 1 & 0.867 & 0.133 & 0.871 & 0.129 & 0.866 & 0.134 \\
\hline 2 & 0.760 & 0.240 & 0.753 & 0.247 & 0.765 & 0.235 \\
\hline 3 & 0.640 & 0.360 & 0.634 & 0.366 & 0.649 & 0.351 \\
\hline \multirow[t]{9}{*}{4} & 0.827 & 0.173 & 0.821 & 0.179 & 0.823 & 0.177 \\
\hline & \multicolumn{2}{|c|}{ Number of latent variables } & 3 & 3 & 5 & 5 \\
\hline & Accuracy & RMSEC $^{\mathbf{a}}$ & 0.779 & 0.779 & 0.143 & 0.143 \\
\hline & & RMSECV $^{b}$ & 0.706 & 0.706 & 0.164 & 0.164 \\
\hline & & RMSEP $^{c}$ & 0.542 & 0.542 & 0.117 & 0.117 \\
\hline & & $\mathbf{R M S} \% \mathbf{R E} \mathbf{E}^{\mathrm{d}}$ & 0.8 & 2.7 & 0.8 & 1.9 \\
\hline & \multicolumn{2}{|l|}{ Sensitivity } & 1.2 & 1.2 & 1.5 & 1.5 \\
\hline & \multicolumn{2}{|c|}{ Analytical sensitivity $^{-1}$} & 1.5 & 0.4 & 1.9 & 0.8 \\
\hline & \multicolumn{2}{|c|}{$\mathbf{R}^{2}$ Prediction } & 0.995 & 0.995 & 0.999 & 0.999 \\
\hline
\end{tabular}

${ }^{\mathrm{a}} \mathrm{RMSEC}$ - the root mean squares regression error.

${ }^{\mathrm{b}} \mathrm{RMSECV}$ - the root mean squares regression error of cross-validation.

${ }^{\mathrm{C}} \mathrm{RMSEP}$ - the root mean squares regression error of prediction.

${ }^{\mathrm{d}} \mathrm{RMS} \% \mathrm{RE}$ - the root mean squares percent relative error.

of latent variables while PLS models are formed by maximal 6 latent variables.

Taking into account the analytical range of the models, acceptable results for sensitivity and analytical sensitivity were observed in the both regressions. Lower values of analytical sensitivity were obtained by the PCR models, while sensitivity is higher in comparison with the results from the PLS models. The sensitivity expressed a minimum concentration difference, therefore has more weight in considering on the suitability of the calibration model. An analytical sensitivity for evaluation of the results was not used, because spectral noise, representing the largest source of errors on the prediction ability of the model, was not taking into account. The models built from the synchronous spectra at $\Delta \lambda=50 \mathrm{~nm}$ provide slightly more suitable results when compared with the models from the spectra recorded at $\Delta \lambda=30 \mathrm{~nm}$.

\section{Conclusions}

A simple method based on synchronous fluorescence spectrometry coupled with MVC was developed for the determination of enantiomeric composition of FLX in complex matrix of biological samples (urine). This affiliation offers cheap and fast option against traditional chiral methods. According to the lowest RMS \%RE values $\Delta \lambda=30$ and $50 \mathrm{~nm}$ were chosen for MVC by PCR and PLS methods. PLS model, constructed from spectral data measured at $\Delta \lambda=50 \mathrm{~nm}$, provides excellent statistical characteristics and appropriate results. The validation of proposed method was carried through the calculation of the figure of merit. The above mentioned fluorescence spectrometry with MVC opens the possibility for the future pharmacokinetics and the pharmacodynamics study of drug in a simple way.

\section{Acknowledgement}

This publication was supported by the Competence Center for SMART Technologies for Electronics and Informatics Systems and Services, ITMS 26240220072, funded by the Research EDDevelopment Operational Programme from the ERDF and the Scientific Grant Agency of the Ministry of Education of Slovak Republic and the Slovak Academy of Sciences VEGA No 2/0033/14.

\section{References}

Ali SM, Asmat F, Maheshwari A, Koketsu M (2005) Il Farmaco 60: 445-449.

Asensi-Bernardi L, Martin-Biosca Y, Fornet-Herrero E, Sagrado S, Medina-Henández MJ (2013) Biomedical Chromatography 27: 377-381.

Douša M, Lehnert P, Adamusová H, Bosáková Z (2013) Journal of Pharmaceutical and Biomedical Analysis 74: $111-116$.

Finn MG (2002) Chirality 14: 534.

Ferré J, Faber NM (2003) Chemometrics and Intelligent Laboratory Systems 69: 123-136. 
Gergely A (2000) Journal of Pharmaceutical and Biomedical Analysis 4: 553-561.

Grodner B, Sitkiewicz D (2013) Psychiatria Polska 3: 511-516.

Horvát P, Gergely A, Noszál B (1997) Talanta 44: 1479-1485.

Konno K, Shinna I, Yui HJ (2013) Journal of Molecular Structure 1035: 260-266.

Kusnir J, Leskova L (2005) Analytical letters 38: 1559-1567.

Lerena A, Dorado P, Berecz R, González A, Norberto M, De La Rubia A (2003) Journal of Chromatography $\mathrm{B}$, Analytical technologies in the biomedical and life sciences 783: 25-31.

Li K, Thompson M, McGregor I (2004) Journal of Chromatography B, Analytical technologies in the biomedical and life sciences 804: 319-26.

Mifsud J, Sqhendo LJ (2012) Journal of Pharmacy and Bioallied Sciences 4: 236-245.

Mohr S, Weiss JA, Spreitz J, Schmid MG (2012) Journal of Chromatography A 1269: 352-359.

Poláček R, Májek P (2015) International Journal of Development Research 5(8): 5240-5244.

Poláček R, Májek P (2016) Chemické listy 110: 18-25.

Poláček R, Májek P, Hroboňova K, Sádecka J (2016) Chirality 28: 319-324.
Rajalahti T, Kvalheim OM (2011) International Journal of Pharmaceutics 417: 280-290.

Ribeiro AR, Afonso GM, Castro PML, Tiritan ME (2013) Environmental Chemistry Letters 11: 83-90.

Shamsipur M, Dastjerdi LS, Haghgoo S, Armspach D, Matt D, Aboul-Enein HY (2007) Analytica Chimica Acta 601: 130-138.

Shen J, Yang J, Heyse W, Schweitzer H, Nagel N, Andert D, Zhu Ch, Morrison V, Nemeth GA, Chen TM, Zhao Z, Ayers T, Choi YM (2014) Journal of Pharmaceutical Analysis 4: 197-204.

Sullivan GR (1978) Topics in Stereochemistry 10: 2874.

Suliman FO, Elbashir AA (2012) Journal of Molecular Structure 1019: 43-49.

Wise BM, Gallagher NB, Bro R, Shaver JM, Windig W, Koch RS (2006) PLS Toolboox 4.0 for use with Matlab $^{\mathrm{TM}}$. Eigenvector Research Inc., Wenatchee.

Wold S, Sjostrom M, Eriksson L (2001) Chemometrics and Intelligent Laboratory Systems 58: 109-130.

Yee L, Wong SHY, Skrinska VA (2000) Journal of Analytical Toxicology 24: 651-655.

Yu L, Wang S, Jiang H, Zhou H, Zeng S (2012) Journal of Chromatography A 1326: 97-104. 